CIENCIAMATRIA

Revista Interdisciplinaria de Humanidades, Educación, Ciencia y Tecnología

Año VI. Vol. VI. N 1 . Edición Especial. 2020

Hecho el depósito de ley: pp201602FA4721

ISSN-L: 2542-3029; ISSN: 2610-802X

Universidad Nacional Experimental Francisco de Miranda (UNEFM). Santa Ana de Coro. Venezuela

Edwin Andersson Zelada Florez

DOI 10.35381/cm.v6i1.387

\title{
La Gestión del talento humano y la Satisfacción laboral en Docentes universitarios
}

Management of human talent and job satisfaction in university teachers

Edwin Andersson Zelada-Florez

edwinzeladaflores@hotmail.com

Universidad Tecnológica del Perú, Lima

Perú

https://orcid.org/0000-0003-1355-2130

Recibido: 05 de septiembre de 2020

Aprobado: 25 de noviembre de 2020 


\title{
CIENCIAMATRIA
}

Revista Interdisciplinaria de Humanidades, Educación, Ciencia y Tecnología

Año VI. Vol. VI. N¹. Edición Especial. 2020

Hecho el depósito de ley: pp201602FA4721

ISSN-L: 2542-3029; ISSN: 2610-802X

Universidad Nacional Experimental Francisco de Miranda (UNEFM). Santa Ana de Coro. Venezuela

Edwin Andersson Zelada Florez

\section{RESUMEN}

La investigación tuvo por objetivo determinar la relación entre la Gestión del talento humano y la satisfacción laboral en docentes universitarios peruanos. Diseño no experimental, correlacional o ex post facto. La población correspondiente a la presente investigación, está conformada por 300 docentes de las diferentes áreas de una universidad privada ubicada en Lima, quienes oscilan entre los 20 a 70 años de edad. A partir de los hallazgos encontrados ( $R h o=0,336$ ); y siendo el valor de $p=0,000<0.05$ ), aceptamos la hipótesis general que establece que existe relación entre la Gestión del talento humano y la Satisfacción laboral en los docentes de la Universidad privada en estudio, 2019, y se asevera que a mayor GTH mayor en la satisfacción en el trabajo. Y se demuestra que, a mayor gestión del talento humano, mayor la satisfacción laboral. Con lo cual se logra el objetivo general de la presente investigación.

Descriptores: Universidad; personal académico docente; satisfacción en el trabajo. (Palabras tomadas del Tesauro UNESCO).

\begin{abstract}
The objective of the research was to determine the relationship between Human Talent Management and job satisfaction in Peruvian university teachers. Non-experimental, correlational or ex post facto design. The population corresponding to this research is made up of 300 teachers from the different areas of a private university located in Lima, who range from 20 to 70 years of age. From the findings found (Rho $=0.336$ ); and being the value of $p=0.000<0.05$ ), we accept the general hypothesis that establishes that there is a relationship between Human Talent Management and Job Satisfaction in the teachers of the private University under study, 2019, and it is asserted that the higher GTH higher in job satisfaction. And it is shown that the greater the management of human talent, the greater the job satisfaction. With which the general objective of the present investigation is achieved.
\end{abstract}

Descriptors: Universities; Academic teaching personnel; Job satisfaction. (Words taken from the UNESCO Thesaurus). 


\section{Edwin Andersson Zelada Florez}

\section{INTRODUCCIÓN}

Los profundos cambios a nivel mundial que ha generado la globalización, han causado que las empresas hoy en día, se vean obligadas a efectuar nuevas prácticas y nuevos ejercicios para incorporarse y mantenerse frente a este imparable dinamismo y que apunten a la satisfacción de las necesidades que van surgiendo como producto de dichas transformaciones. Por ello las labores empresariales han tenido que redimensionarse para permanecer en el mercado y para ser más competitivas, de acuerdo con la naturaleza de los nuevos retos (económicos, financieros, tecnológicos, políticos, sociales, culturales, entre otros), en donde el espacio dirigido a la Gestión del Talento Humano, no es ajeno a estos desafíos, hoy en día el papel de éste en la organización se está revalorando, concibiéndose como un garante con compromisos múltiples abocados a reorientar funciones que permitan alcanzar objetivos y metas empresariales, dentro de las cuales destaca sobremanera la satisfacción laboral.

En tal sentido y considerando que, desde hace un tiempo para acá, se viene considerando que él personal dentro de las organizaciones es el generador del éxito, el protagonista indispensable, y la ventaja competitiva que debe ser fortalecida para enfrentar los nuevos retos de la globalización, es que hemos procedido a señalar algunos puntos importantes que analizan con más detalle, la realidad de este sector y para lo cual hemos enfatizado más en el sector de nuestra unidad de análisis: Las casas de estudio a nivel superior.

En primer lugar, señala Cuentas (2018), que la sociedad actual, se caracteriza por la búsqueda continua de ciencia y conocimientos, la cual demanda de las universidades, una renovación de la gestión, donde las personas que producen e imparten conocimientos sean el centro, es decir sean la finalidad principal de una gestión del capital humano. 
Por tanto, en las organizaciones dedicadas a la educación superior, como todas en general, gestionar la recursos humanos es lograr que muchos factores, desde la infraestructura, el estilo de liderazgo, las comunicaciones, las relaciones interpersonales, la estructura organizacional, los procesos evaluadores, la capacitación, el desarrollo del itinerario de carrera y todo el ciclo de la gestión de personas, confluya para que los docentes desarrollen identidad con la institución, descubran el sentido de su trabajo, valoren su trabajo, se sientan motivados para lograr que sus estudiantes aprendan, sientan satisfacción personal con sus logros profesionales y también reciban el reconocimiento de pares, directivos, estudiantes y apoderados (Celis, 2017).

Considerando que es la universidad quien tiene a cargo el llevar el conocimiento más avanzado a sus entornos sociales, construir capacidades en las personas y colocarlas a la altura de la demanda de los tiempos actuales y cambiantes (Unión de Universidades de América Latina y el Caribe (UDUAL), 2019). Para alcanzar su objetivo principal la universidad debe contar con profesores e investigadores, como un recurso que debe ser gestionado para enrumbar a la construcción de conocimientos necesarios que demanda la sociedad (Aguilera \& González, 2018). Lo cual se logra a través de un proceso llamado gestión del talento humano, el cual según Chávez (2018), es la clave para alcanzar la armonía entre las demandas requeridas para el desarrollo del país, los aportes que construyen los profesores e investigadores universitarios.

En ese sentido, el futuro exitoso de las universidades, que tienen la función de formar profesionales competentes, depende de su capacidad de convertir en ventaja su recurso más preciado: El capital humano. Por consiguiente, la universidad deberá ser capaz de potenciar y estimular todas las habilidades necesarias en los miembros de la institución que le permitan desarrollar una serie de habilidades y competencias destinadas a generar más conocimiento y que este repercuta en beneficio de la institución, por consiguiente, del país (Dădârlat \& Dumitraşcu, 2015). 
A nivel internacional, un estudio en los Estados Unidos demostró que para el 2014, se proyectaba en las universidades como en demás instituciones profesionales un crecimiento del 34,3\% (Bureau of Labor Statistics, 2005, citado por (Sabharwal \& Corley, 2009). Por lo tanto, las cuestiones de satisfacción, retención, y la persistencia han sido cada vez más importantes para los administradores universitarios y la política educativa. Por lo que la necesidad de estudiar la satisfacción del profesorado en las universidades también se deriva del hecho de que las estructuras sociales de la educación superior están cambiando con el tiempo. Siguiendo con Estados Unidos, encontramos que, para las décadas venideras, cada vez será más probable que los profesores se jubilen y sean reemplazados por profesores jóvenes especialmente mujeres, minorías infrarrepresentadas o académicos nacidos en el extranjero, lo cual requerirá que los administradores universitarios se ocupen de cuestiones relacionadas a la satisfacción laboral del profesorado en una variedad de dimensiones personales y profesionales (Sabharwal \& Corley, 2009).

En dicho estudio se demostró que la satisfacción de los docentes varía según género y disciplinas, en todos los campos los hombres tienen niveles significativamente más altos de satisfacción laboral en general y niveles significativamente más altos de satisfacción con la seguridad laboral. Por su lado las mujeres con mayor satisfacción son las del campo de la salud (media $=3,41$ ), seguido por ciencias (media $=3,40$ ), ciencias sociales (media $=3,38$ ) y por último la ingeniería (media $=3,31$ ). Mientras que en los hombres, se mostraban igual de satisfechos tanto en las ciencias sociales, ingeniería y los campos de salud (media $=3,47$ ), donde señalaban que estos consiguen mayores oportunidades de progreso que las mujeres, los hombres demostraron tener salarios más altos que las mujeres, especialmente los docentes del área de la ingeniería, por lo que estas discrepancias influyen en los niveles de satisfacción de las mujeres respecto a sus 


\section{Edwin Andersson Zelada Florez}

salarios anuales; y en cuanto a los beneficios laborales las mujeres señalaron: que están más satisfechas que los hombres en este aspecto, y dentro del ámbito de independencia laboral, los profesores masculinos estaban más satisfechos que los miembros femeninos. Por su parte al evaluar el lugar de trabajo los miembros masculinos tenían más probabilidades de estar satisfechos, y respecto a la responsabilidad laboral, las mujeres demostraron mayor satisfacción. En este sentido este autor sugirió que se debe considerar una medida de satisfacción laboral general para implementar políticas porque muchas veces el cambio puede ser engañoso. Otro estudio realizado por Seifert \& Umbach (2008), señala que en USA las características demográficas tienen efectos sobre las dimensiones de la satisfacción laboral, especialmente el género, donde las mujeres constantemente son menos satisfechas, y el efecto varió en la mujer según la disciplina, otros factores son la raza o etnia quien tiene efectos mixtos en las dimensiones de trabajo. Por lo que las diversas características demográficas son predictores de la satisfacción laboral en las universidades de este país.

En Europa, específicamente en Lituania, la satisfacción laboral de los docentes en instituciones de educación superior, son influenciadas especialmente por la efectividad organizacional, el liderazgo en la cultura, el estilo de liderazgo, el comportamiento de la dirección, la gestión universitaria, el salario de las universidades, las oportunidades de promoción y las condiciones laborales tienen las impacto en la satisfacción laboral siendo temas que están en aumento en las últimas décadas, siendo el liderazgo un concepto que ha venido evolucionado, producto de los cambios en la demografía, globalización, tecnología, y prácticas laborales; y que es fundamental ya que actualmente las universidades compiten por atraer estudiantes (Raimonda \& Modesta, 2016).

En Asia, específicamente en la India, un estudio de Bholane \& Suryawanshi (2015), sostiene que la satisfacción laboral de los docentes universitarios es fundamental y necesaria, lo cual debe ser alcanzando a través de una eficaz gestión de los recursos para que estos logren prestar al máximo el servicio a la sociedad. En un estudio llevado 


\section{Edwin Andersson Zelada Florez}

a cabo también en la India por Maharashtra, los docentes opinaron que se encontraban satisfechos con su profesión, el $97 \%$ disfrutan de la seguridad laboral, solo el $6 \%$ deseaba cambiar de profesión, el fenómeno psicológico del deseo, la autonomía laboral, la creatividad, sentido de logro, la carga de trabajo adecuada, la sensación de frescura son algunos de los elementos que mayor explican la satisfacción laboral de los académicos universitarios, por lo que se puede decir, que existen una notoria relación entre los elementos del trabajo y la satisfacción del profesorado.

En América Latina, se ha observado en las últimas décadas, grandes esfuerzos en la administración de la academia tanto públicas como privadas, pese a ser concebida la universidad como uno de los fenotipos organizacionales de mayor complejidad, ya que cada grupo afín (docentes, estudiantes, investigadores, administrativos) tienen distintos intereses, fines y racionalidades, que en muchos casos son incompatibles y opuestas, lo cual conduce a dificultades en la toma de decisiones, por ejemplo en las universidades argentinas, los factores que explican una mayor complejidad en el sistema educativo, y que por ende afecta de una u otra manera es la disminución de la dedicación horaria a la enseñanza, siendo la mayor ocupación el ejercicio liberal, así como también existe relación con la edad, donde se observa que la satisfacción laboral de los docentes va disminuyendo con el pasar del tiempo, teniendo un mínimo alrededor de los 46 años, y justo en este punto retoma a una tendencia ascendente; $y$ los factores externos de mayor consideración para la satisfacción del docente son las condiciones de empleo (Pujol, 2016).

En México, el 26,9\% de los profesores universitarios presentan insatisfacción laboral, siendo los factores de mayor influencia la compensación y las condiciones de trabajo, sumado al agobio propio de sus funciones, y el cumplimiento que deben dar también a las labores administrativas (Aguilar, Magaña, \& Guzmán, 2015). En Chile, un estudio demostró que el $95 \%$ de los docentes se sientes satisfechos laboralmente, un $80 \%$ percibe un alto nivel de clima organizacional, y quienes más se sienten satisfechos son 


\section{Edwin Andersson Zelada Florez}

aquellos docentes que tienen entre 15 a 29 años de antigüedad en la universidad y los que trabajan menos de 40 horas a la semana (Cáceres, y otros, 2017).

En Venezuela, la Gestión del Talento Humano en la universidad ha permitido mejoras cuando aplican capacitaciones en el área de TIC, donde el 61,75\% presentan deseos de aprender, mientras que un $22,42 \%$ manifestaron no encontrar la utilidad para involucrar la herramienta virtual en el proceso de enseñanza y aprendizaje. Se aprecia que en la mayoría de las gestiones del capital humano en las instituciones de educación superior, a nivel tanto de pregrado como de postgrado han tenido que redefinir su rol y orientar la planificación a nuevas estrategias de naturaleza tecnológica, infraestructura tecnológica y virtual, establecimiento de línea de investigación, relación de toda la comunidad universitaria, para cooperar con la satisfacción en el recinto universitario no solo de los docentes sino de los estudiantes (Ibáñez \& Castillo, 2008).

En el Perú, en un trabajo realizado para una universidad, se demostró que el $71 \%$ de los docentes, no conocen incentivos otorgados por las instituciones, y un $29 \%$ señalo que el único incentivo conocido por ellos y aceptado por los directivos es el reconocimiento personal y moral por sobresalir en su catedra, y el $100 \%$ coinciden que no existe un sistema formalizado de incentivos que funcione como impulsor de la inversión del capital humano en la institución. Los directivos expresan que están conformes con la remuneración que es otorgada, pero ninguno señala que le es atractiva (Cuentas, 2018). Este panorama es muy similar a lo que se observa y demuestra en muchas universidades del país, pues la gestión llevada, no prioriza el capital humano como elemento fundamental en el desarrollo, es decir no son considerados a los docentes como inversores de capital humano, por tanto, carecen o dificultan su ventaja competitiva. Lo cual como señala Alles (2007), en las instituciones del conocimiento, los docentes representan una ventaja competitiva, siendo ellos una herramienta diferenciadora, son el contacto directo con los usuarios, es decir con los estudiantes y afines, quienes buscan en las universidades productos de calidad (educación de calidad). 


\section{Edwin Andersson Zelada Florez}

En el caso de la universidad privada que se consideró como la unidad de análisis del presente trabajo, tuvo una situación muy similar a los problemas encontrados en otras universidades del país, a ello se le suma la incertidumbre vivida por el cambio de autoridad y gestión ocurrido en el 2012 cuando un reconocido grupo empresarial, adquirió primeramente la mitad de sus acciones y luego el 100\% del accionariado en el 2017 (Gestión, 2012; Perú Retail, 2017). Lo cual generó cambios significativos en la manera que se venía gestionando los recursos humanos y financieros.

Por lo que surgió en los docentes mucha incertidumbre, ya que muchas de las condiciones institucionales y laborales que hasta ese momento permitían desarrollar una práctica pedagógica amena, pasó a ser modificada (carga horaria, lugares de descanso, atención en los servicios básicos, etc.), lo cual trajo como consecuencia una inmediata insatisfacción laboral, así como otros problemas ligado a las condiciones laborales, destacando más la desmotivación de los docentes.

Era muy notorio y por ello se considera que en gran parte los problemas generados fueron producto de un cambio en la gestión de recursos humanos, lo cual atendió a nuevas normas que no consideraron las maneras en cómo se venía llevando a cabo los procesos, por lo cual los docentes no fueron participes del cambio de gestión, sintiéndose poco incentivados y valorados, viéndose reflejado a la larga en un aumento del índice de rotación de personal y también en su desempeño docente.

Por lo cual, la reforma de la gestión universitaria ocurrida en este periodo de cambio de administración no fue exitosa, pues el desafío que se tenía para el momento implicaba más que un cambio de condiciones laborales, precisaba de capacitación e integración de la nueva administración junto con el personal. Como sostiene Pérez (2011), la gestión de recursos humanos dentro de los paradigmas actuales de las universidades, deben estar enfocados en orientar los recursos científicos, tecnológicos y financieros de tal manera que se logren alcanzar los objetivos deseados por la institución y la sociedad en general. Como explica Cuentas (2018), dentro de dicho paradigma, la valorización de los recursos 
humanos a través de la formación del personal y la innovación permanente con la administración de los recursos técnicos son la clave para lograr la excelencia y eficiencia organizacional.

La investigación tuvo por objetivo determinar la relación entre la Gestión del talento humano y la satisfacción laboral en docentes universitarios peruanos.

\section{MÉTODO}

El presente trabajo es de tipo básico. Según Vargas (2009), también se le conoce como una investigación fundamental exacta o investigación pura. Esta se centra en el objeto de estudio sin llegar a considerar una aplicación inmediata sobre el problema, sin embargo, sus resultados son las bases para nuevos productos científicos. Como expresa Maya (2014), busca el progreso científico, persiguiendo generalizaciones con vistas al desarrollo de hipótesis de carácter universal, enmarcada según el diseño No experimental de forma transeccional - correlacional. Hernández, Fernández, \& Baptista (2014), expresan que las investigaciones no experimentales son: "Estudios que se realizan sin la manipulación deliberada de variables y en los que solo se observan los fenómenos en su ambiente natural para después analizarlos". (p.152). Los diseños transeccionales - correlacionales describen relaciones entre dos o más categorías, conceptos o variables en un momento determinado.

Dentro de la investigación no experimental, corresponde al tipo correlacional o ex post facto, el cual según Tamayo (2007), porque se busca determinar el grado en el cual las variaciones en uno o varios factores están relacionadas con la variación en otro u otros factores. Como menciona McMillan \& Schumacher (2005), valora las relaciones entre dos o más fenómenos, y por lo generar, implica una medida estadística de relación, llamada correlación, siendo esta una afirmación sobre el grado de asociación entre las variables de interés. 


\section{Edwin Andersson Zelada Florez}

En cuanto a la población correspondiente a la presente investigación, está conformada por 300 docentes de las diferentes áreas de una universidad privada ubicada en Lima, quienes oscilan entre los 20 a 70 años de edad. Y la muestra es del tipo no probabilístico compuesta por 169 docentes según cálculo estadístico para población finita, quienes proporcionaron la información necesaria para el estudio. Y el muestreo utilizado es el de la Tómbola, también conocido como sorteo o rifa, que consiste en enumerar todos los elementos muéstrales del uno al número "n", después se hacen fichas, uno por cada elemento, se revuelven en una caja y se van sacando " $n$ " números de fichas según el tamaño de la muestra. Los números elegidos al azar conformaran la muestra (López, 2004).

Para recoger los datos de la variable Gestión del talento humano y Satisfacción Laboral, se utilizó la técnica de la encuesta, la cual, según Hernández, et al. (2014), es una técnica la recolección sistémica de información de manera oral o escrita sobre datos de un grupo o muestra de individuos o en relación a la opinión de los encuestados sobre un tema específico. El instrumento utilizado para recoger datos de las variables, fue un cuestionario, según Arias (2006), el cuestionario es "la modalidad de encuesta que se realiza de forma escrita mediante un instrumento o formato en papel contentivo de una serie de preguntas. Se le denomina cuestionario auto administrado porque debe ser llenado por el encuestado, sin intervención del encuestador" (p.74).

Para el cual se consideró todos los aspectos relacionados con las dimensiones de ambas variables en estudio, y que permitieron demostrar los objetivos establecidos en el presente trabajo. Este instrumento está conformado por una serie de afirmaciones dirigidas a la muestra, utilizando la escala psicométrica de Likert (nunca, casi nunca, algunas veces, casi siempre, y siempre). La Escala de Likert, permite una medición multidimensional en una escala ordinal que permite medir una actitud y el comportamiento consecuente con ésta. Se basa en la premisa según la cual la intensidad de las apreciaciones, opiniones o imágenes de un individuo puede ser escalada dentro 


\section{Edwin Andersson Zelada Florez}

de ciertas categorías. Así, en lugar de preguntar abiertamente a las personas su opinión acerca de un tema, ellas son enfrentadas a unos enunciados o afirmaciones cuyos contenidos, por sus características, los colocan ante la posibilidad de tener que manifestar sus opiniones dentro de una escala de categorías posibles de respuestas (Aigneren, 2003).

Dicho instrumento fue validado a través de juicio de expertos, considerando que la validez, como señala Hernández, et al. (2014), se refiere al grado en que un instrumento realmente mide la variable en la cual se pretende medir, es decir, que sus resultados deben contestar las preguntas formuladas y no otro asunto.

La confiabilidad o consistencia interna de los datos del cuestionario aplicado para medir ambas variables, fue estimado mediante el estadístico de fiabilidad "Alfa de Cronbach" para cada uno de sus dimensiones, tal como señala Donald, Cheser, \& Razavieh (1989), este fundamenta el grado de uniformidad con que los instrumentos cumplen con la finalidad. Estos fueron procesados utilizando el software estadístico SPSS 25.0, y fueron interpretados considerando como criterios generales los propuestos por George \& Mallery (2003), quienes sugieren las siguientes recomendaciones para evaluar los coeficientes de alfa de Cronbach (Tabla 1):

\section{Tabla 1.}

Criterio general para interpretar el coeficiente Alfa de Cronbach.

\begin{tabular}{ll}
\hline Coeficiente alfa & Descripción \\
\hline Coeficiente alfa $>.9$ & Excelente \\
Coeficiente alfa $>.8$ & Bueno \\
Coeficiente alfa $>.7$ & Aceptable \\
Coeficiente alfa $>.6$ & Cuestionable \\
Coeficiente alfa $>.5$ & Pobre \\
Coeficiente alfa $<.5$ & Inaceptable \\
\hline
\end{tabular}

Fuente: George \& Mallery (2003, p. 231). 


\section{Edwin Andersson Zelada Florez}

Obteniendo el valor de 0,802 para la variable gestión del talento humano, para los 10 ítems, lo cual significa según George \& Mallery (2003), es una confiabilidad buena; y un 0,827 para la variable satisfacción laboral, lo cual según criterio de George \& Mallery (2003), representa una confiabilidad buena.

\section{RESULTADOS}

A partir de los hallazgos encontrados ( $R h o=0,336)$; y siendo el valor de $p=0,000<0.05$ ), aceptamos la hipótesis general que establece que existe relación entre la Gestión del talento humano y la Satisfacción laboral en los docentes de la universidad en estudio en el año 2019, y se asevera que a mayor GTH mayor en la satisfacción en el trabajo, se demuestra que a mayor gestión del talento humano, mayor la satisfacción laboral. Con lo cual se logra el objetivo general de la presente investigación, se puede indicar que mayor responsabilidad social, mayor satisfacción laboral.

\section{DISCUSIÓN}

La importancia de la gestión del talento humano como determinante del éxito, la satisfacción y la rentabilidad de las organizaciones, por ello es fundamental que, en los estudios sobre el comportamiento organizacional, se evalué la satisfacción laboral, siendo de importante interés no solo a nivel personal de cada colabores sino también para la organización. Tal es el caso de Dender (2016), quien expuso que a través de la gestión del talento humano se pueden mejorar los procesos en las áreas académicas y administrativas, lo que ayuda entre otras cosas, a que los docentes, personal adquieran competencias y se sientan satisfechos, viéndose reflejado en la calidad de la educación. 


\section{Edwin Andersson Zelada Florez}

También concuerda con Bernal (2015), quien señala que por medio de la GTH se mejoran los procesos administrativos, ya sea la planeación, reclutamiento, capacitación o evaluación, de esta manera el empleado estará más comprometido con los objetivos empresariales; o como obtuvo Guere (2019), la gestión del talento humano tiene correlación alta con el control de la calidad del servicio, en la admisión del personal, en las capacidades del personal, en las compensaciones laborales en las universidades, y todo ello puede ser medido a través de la satisfacción al cliente como medida de la calidad del servicio ofrecido, información que es de suma importancia para la competitividad de la empresa. No obstante, apoya a los resultados encontrados por Reyes (2018), donde señala que es determinante para el área de gestión del talento humano, conocer a fondo la formación académica, el compromiso y las habilidades blandas para poder inferir sobre las propias necesidades de los docentes, y de esta manera acertar las estrategias que permitan lograr una satisfacción en el trabajo. Como sostiene Tinoco (2017), el desarrollo del talento humano en la universidad debe ser reflejo su cultura organizacional, de sus valores, y es una ventaja competitiva.

\section{CONCLUSIÓN}

A partir de los hallazgos encontrados ( $R h o=0,336$ ); y siendo el valor de $p=0,000<0.05$ ), aceptamos la hipótesis general que establece que existe relación entre la Gestión del talento humano y la Satisfacción laboral en los docentes de la Universidad privada en estudio, 2019, y se asevera que a mayor GTH mayor en la satisfacción en el trabajo. Y se demuestra que, a mayor gestión del talento humano, mayor la satisfacción laboral. Con lo cual se logra el objetivo general de la presente investigación.

\section{REFERENCIAS CONSULTADAS}

Aguilera, L., \& González, C. (2018). La capacidad propositiva de la universidad y el desarrollo local. Ponencia al Congreso Universidad 2018. La Habana. 


\section{Edwin Andersson Zelada Florez}

Aguirre, C., Andrade, M., \& Castro, A. (2005). Desarrollo de un Instrumento de Variables que Podrían Influir en la Satisfacción Laboral de Trabajadores de la Construcción en Santiago de Chile. Revista de la Construcción, 4(1), 81-90.

Aigneren, M. (2003). Diseños cuantitativos: análisis e interpretación de la información. Colombia: Fondo Editorial CEO.

Alles, A. (2007). Comportamiento organizacional: cómo lograr un cambio cultural a través de la Gestión por competencias. Buenos Aires: Granica.

Arias, F. G. (2006). El Proyecto de Investigación. Introducción a la Metodología Científica (5a. ed.). Caracas: Episteme.

Bernal, J. (2015). Administración de Recursos Humanos y Compromiso Laboral de los Empleados del Corporativo Adventista de Montemorelos. (Tesis de maestría). Universidad de Montemorelos, Montemorelos, México. Obtenido de https://n9.cl/amotx

Bholane, K., \& Suryawanshi, J. (2015). A Study of Job Satisfaction of University Teachers in Maharashtra State. Management Today, An International Journal of Management Studies, 5(4), 192-195. doi:http://dx.doi.org/10.11127/gmt.2015.12.07

Cáceres, P., Beiio, N., Bermúdez, N., Burgos, F., Fuentealba, M., \& Padilla, A. (2017). Satisfacción Laboral y su Relación con el Clima Organizacional en Funcionarios de una Universidad Estatal Chilena. Revista Ciencia \& Trabajo, 19(58), 7-13. Obtenido de https://n9.cl/1pc6o

Celis, M. (2017). Gestión de Recursos Humanos en la Escuela: un Desafío Permanente. Chile: Gobierno de Chile- Fundación Chile. Obtenido de https://n9.cl/f1z3

Chávez, L. (2018). La gestión del talento humano como estrategia empresarial. Perspectiva, 19(4), 553-558.

Cuentas, S. (2018). La gestión del capital humano en una unidad académica de educación superior. Revista Educación, 57-72. Obtenido de http://www.scielo.org.pe/pdf/educ/v27n53/a04v27n53.pdf 
Dădârlat, A., \& Dumitraşcu, D. (2015). The role and importance of human capital in contemporary organizations. Review of Management \& Economic Engineering, 14(1), 78-86. Obtenido de https://n9.cl/fkih

Dender, R. (2016). Gestión del Talento Humano por Competencias de los Docentes de la Carrera de Ingeniería Eléctrica de la Universidad Técnica Luis Vargas Torres. (Tesis de maestría). Pontificia Universidad Católica del Ecuador, Esmeraldas, Ecuador. Obtenido de https://n9.cl/g0ma3

Donald, A., Cheser, L., \& Razavieh, A. (1989). Introducción a la investigación pedagógica. México: McGraw-Hill.

George, D., \& Mallery, P. (2003). SPSS for Windows step by step: A simple guide and reference. 11.0 update (4thed.). Boston: Allyn \& Bacon.

Gestión. (2012). Grupo Interbank compró la Universidad Tecnológica del Perú. Obtenido de https://n9.cl/ylun

Guere, H. (2019). Gestión del Talento Humano con Relación al Control de Calidad de Servicio de la Empresa Valuaciones Maneras SAC, 2019. (Tesis de grado). Universidad San Ignacio de Loyola, Lima, Perú. Obtenido de https://n9.cl/3luv

Hernández, R., Fernández, C., \& Baptista, P. (2014). Metodología de la Investigación (6a. ed.). México: McGraw-Hill.

Ibáñez, N., \& Castillo, R. (2008). Gestión del capital humano en el contexto postmoderno de la educación superior y el uso de las tecnologías de información y comunicación. Revista Latinoamericana de Estudios Educativos (Colombia), 4(1), 105-128. Obtenido de https://www.redalyc.org/pdf/1341/134115209008.pdf

Maya, E. (2014). Métodos y técnicas de investigación. México: Universidad Nacional Autónoma de México.

McMillan, J., \& Schumacher, S. (2005). Investigación educativa (5a. ed.). Madrid: Pearson Educación

Pérez, A. (2011). Fundamentos de la gestión del conocimiento en la universidad. Revista Gestión Universitaria, 3(2). Obtenido de https://n9.cl/ek7ft

Perú Retail. (2017). Intercorp a través de Nexus Group adquiere totalidad de acciones de UTP. pág. Perú Retail. Obtenido de https://n9.cl//88k 
Pujol, L. (2016). Satisfacción Laboral en docentes universitarios: medición y estudio de variables influyentes. Redu Revista de docencia universitaria, 14(2), 261-292. Obtenido de https://dialnet.unirioja.es/descarga/articulo/5765940.pdf

Raimonda, A., \& Modesta, M. (2016). Leadership style and job satisfaction in higher education institutions. International Journal of Educational Management, 30(1), 140-164. doi:https://doi.org/10.1108/IJEM-08-2014-0106

Reyes, Á. (2018). El talento humano de los profesionales de la información y los servicios en la biblioteca de la Universidad de Piura en el Perú. (Tesis de grado). Universidad Nacional Mayor de San Marcos, Lima, Perú. Obtenido de https://n9.cl/3cnr

Sabharwal, M., \& Corley, E. (2009). Faculty job satisfaction across gender and discipline. The Social Science Journal, 46(3), 539-556. Doi: 10.1016 / j.soscij.2009.04.015

Seifert, T., \& Umbach, P. (2008). The Effects of Faculty Demographic Characteristics and Disciplinary Context on Dimensions of Job Satisfaction. Res High Educ (49), 357381. Doi:10.1007/s11162-007-9084-1

Tamayo, M. (2007). El proceso de la investigación científica. México: Limusa. (4a. ed.). México: Limusa.

Tinoco, R. (2017). El desarrollo del talento humano en la Universidad Corporativa Intercorp. (Tesis de maestría). Pontificia Universidad Católica del Perú, Lima, Perú. Obtenido de https://n9.cl/j7bg0

Unión de Universidades de América Latina y el Caribe (UDUAL). (2019). Declaración de la Asamblea General de la UDUAL. Declaración de la Asamblea General de la UDUAL, 28 y 29 de noviembre de 2019. Lima, Perú: Universidad Ricardo Palma.

Vargas, Z. (2009). La investigación aplicada: una forma de conocer las realidades con evidencia científica. Revista Educación, 33(1), 15-165. Obtenido de https://n9.cl/2taq 
CIENCIAMATRIA

Revista Interdisciplinaria de Humanidades, Educación, Ciencia y Tecnología

Año VI. Vol. VI. N¹. Edición Especial. 2020

Hecho el depósito de ley: pp201602FA4721

ISSN-L: 2542-3029; ISSN: 2610-802X

Universidad Nacional Experimental Francisco de Miranda (UNEFM). Santa Ana de Coro. Venezuela

Edwin Andersson Zelada Florez

@2020 por el autor. Este artículo es de acceso abierto y distribuido según los términos y condiciones de la licencia Creative Commons Atribución-NoComercial-Compartirlgual 4.0 Internacional (CC BY-NC-SA 4.0)

(https://creativecommons.org/licenses/by-nc-sa/4.0/). 